\title{
Pygmy and giant resonances: connecting the nuclear structure to stellar as- trophysics
}

\author{
Nadia Tsoneva ${ }^{1, *}$ \\ ${ }^{1}$ ELI-NP, Horia Hulubei National Institute for Physics and Nuclear Engineering, 30 Reactorului Street, RO-077125, Bucharest- \\ Magurele, Romania
}

\begin{abstract}
Systematic theoretical investigations of dipole response in neutron-rich nuclei of importance for day-one photonuclear experiments at ELI-NP are presented. The calculations are performed in advanced microscopic theory based on energy-density functional and three-phonon quasiparticle-phonon model. The model basis accounts for a large range of nuclear excitations with different spin and parity. In the focus of the studies are electric and magnetic dipole modes with energies up to $25 \mathrm{MeV}$. Of special interest are pygmy dipole and giant dipole resonances. The impact of different low-energy excitations and in particular of the PDR on radiative neutron-capture reaction cross sections in nuclei of key importance for nucleosynthesis is investigated.The obtained results are compared to available experimental data.
\end{abstract}

\section{Introduction}

Recently, new aspects of nuclear dynamics below and around the neutron threshold in nuclei with small and moderate neutron excess has been explored in our studies of low-energy electric and magnetic dipole and quadrupole excitations [1]. The dynamics of such nuclear systems is very sensitive to the nuclear symmetry energy and its density dependence which on the other hand is imprinted in a static mean-field observable - the neutron-skin thickness [2-4]. Furthermore, the presence of a neutron skin may influence nuclear response on external electromagnetic fields. An observable which could be sensitive to induced skin effects on nuclear excitations, especially at low-energies, is the nuclear dipole polarizibality [2-4]. In the past years, new modes of nuclear excitation closely connected with the skin phenomena in atomic nuclei- the pygmy dipole resonance (PDR) [5-12] and its natural extension at higher multipolarities - the pygmy quadrupole resonance (PQR) [13-15] have been observed and investigated. Of our special interest is the description of fine and gross spectral features of these new modes of nuclear excitations and the relation between the pygmy resonances and the neutron skin thickness [1].

Presently, a direct method to extract experimentally the neutron skin thickness from the PDR does not exist. This could be related to the fact that at excitation energies below the neutron separation energy the pygmy resonances of different multipolarity coexist with a variety of modes, such as the tail of the giant resonances and multi-phonon excitations. However, distinction between pygmy and other modes could be obtained theoretically in our microscopic theory $[1,4,10,16]$. In this connection, we have studied recently theoretically and experimentally spectral distributions of electric and magnetic dipole modes up to giant resonance energies in ${ }^{206} \mathrm{~Pb}$ [4]. Such studies are able to provide information on fine and gross microscopic features of the nuclear $\gamma$-strength function as well as they allow for precise determination of dipole polarizability which could be further referred to the nuclear skin thickness.

In these and other previous investigations we show the importance of the PDR for understanding of fundamental properties of the nucleus and nuclear matter such as nuclear skin thickness and symmetry energy as well as for nuclear astrophysics and nucleosynthesis of heavier elements in the universe [4, 17-19]. In particular, our microscopically obtained $\gamma$-strength functions could be implemented in a statistical reaction model [17] and used for estimations of nuclear reaction rates and astrophysical applications $[4,18,19]$. Recently, we systematically investigated the contribution of the PDR to radiative capture cross sections for $10 \div 100 \mathrm{keV}$ incident neutrons in $\mathrm{N}=50$ isotones [18]. The results obtained within energy-densityfunctional+quasiparticle-phonon-model (EDF+QPM) approach [18] indicate that they are correlated with the neutron skin thickness. In particular, the largest PDR impact, of the order of $\approx 50 \%$, is observed for the ${ }^{85} \mathrm{Kr}(\mathrm{n}, \gamma)^{86} \mathrm{Kr}$ reaction, where the target nucleus ${ }^{85} \mathrm{Kr}$ has the largest neutron excess in comparison with the other considered $\mathrm{N}=50$ isotones. The effect smoothly decreases with the decrease of the N/Z ratio towards ${ }^{91} \mathrm{Mo}$ and its contribution to the ${ }^{91} \mathrm{Mo}(\mathrm{n}, \gamma){ }^{92}$ Mo reaction cross section is of the order of $10 \%$. Similar results are also found in the examination of Maxwellian-averaged cross sections at the thermal energy of $\mathrm{kT}=30 \mathrm{keV}[18]$.

\footnotetext{
*e-mail: nadia.tsoneva@eli-np.ro
} 
Furthermore, a common observation in these studies is, that the quasiparticle-random-phase-approximation (QRPA) is not sufficient to describe the nuclear excitations below the neutron threshold and hence, the radiative capture cross sections at astrophysical energies. The systematic comparison between EDF + QRPA and the threephonon $\mathrm{EDF}+\mathrm{QPM}$ calculations in $\mathrm{N}=50$ nuclei shows that the behavior of the low-energy dipole strength is influenced by the competition between static and dynamical effects. The first one is related to the mean field and the pure PDR associated with neutron skin oscillations, while the second one represents the coupling of the single-particle states with a more complex excitations related to core polarization and giant dipole resonance (GDR). These effects lead to the redistribution and fragmentation of the lowenergy dipole strength. Consequently, the three-phonon QPM calculations indicate that the obtained within the QRPA radiative capture cross sections in $\mathrm{N}=50$ isotones are underestimated by a factor of about two.

It should be mentioned that $\mathrm{N}=50$ nuclei are of great importance for the nucleosynthesis and astrophysics. A typical case is the nucleus ${ }^{85} \mathrm{Kr}$ which is known as a "branching-point" nucleus of the s-process of nucleosynthesis and such nuclei are of particular interest because they can provide information on the neutron flux and temperature at the s-process site $[18,19]$.

Another interesting example is the neutron-rich ${ }^{206} \mathrm{~Pb}$ $(\mathrm{N} / \mathrm{Z}=1.51)$ nucleus. The latter is expected to have a robust neutron skin, and as a consequence additional lowenergy dipole strength correlated with the skin dynamics. Furthermore, the presence of PDR mode in ${ }^{206} \mathrm{~Pb}$ might affect the ${ }^{205} \mathrm{~Pb}$ radiative neutron capture cross section, a reaction of relevance to the destruction of ${ }^{205} \mathrm{~Pb}$ during the s-process [4]. It is well known that the radionuclide ${ }^{205} \mathrm{~Pb}$ with a half-life of $15.3 \mathrm{Myr}$ may be of significant cosmochemical interest due to its pure s-process nature. Furthermore, it could provide key information on the formation of the solar system [4].

In this manuscript, the role of the PDR for the ${ }^{205} \mathrm{~Pb}$ neutron-capture process is examined and discussed in detail. Furthermore, EDF+three-phonon QPM results on the fine and gross spectral features of E1 and M1 excitations up to GDR energies are presented and compared to experimental data [4].

\section{The theoretical approach}

Our theoretical approach is based on EDF theory and three-phonon QPM theory [6, 8]. In the last years the method was implemented in the description of the structure of nuclear electric and magnetic excitations in the PDR region and it was found very successful for description of the low-energy $\gamma$-strength functions and more particularly of the PDR [1]. An important advantage of the $\mathrm{EDF}+\mathrm{QPM}$ approach is the description of the nuclear excitations in terms of QRPA phonons as a building blocks of the three-phonon QPM model space [20,21] which provides a microscopic way to multi-configuration mixing. Thus, the EDF+QPM allows for sufficiently large configuration spaces which is required for a unified description of low-energy single- and multi-particle states and also for the GDR energy region.

The model Hamiltonian is given by

$$
H=H_{M F}+H_{\text {res }},
$$

where $H_{M F}$ is a mean-field part and $H_{r e s}$ stands for the residual interaction.

The mean-field (MF) part $H_{M F}$ is treated by selfconsistent Skyrme Hartree-Fock-Bogoliubov (HFB) theory described in $[1,22]$. The pure HFB picture is in fact extended beyond MF by dynamical self-energies, hence incorporating a more detailed spectral description of nuclear spectra. The procedure allows us to account in a selfconsistent manner for nuclear binding energies and other ground-state properties of nuclei like the charge radii and the neutron skin thickness and to reproduce precisely the results obtained from Skyrme SLy4HFB calculations [8] and available experimental data [1, 22].

The nuclear excited states are calculated with a residual interaction which is based on the QPM formalism [20]:

$$
H_{\text {res }}=H_{M}^{p h}+H_{S M}^{p h}+H_{M}^{p p},
$$

where effective interactions are implemented to account for the interaction between the quasiparticles. The terms $H_{M}^{p h}, H_{S M}^{p h}$ and $H_{M}^{p p}$ are taken as a sum of isoscalar and isovector separable multipole and spin-multipole interactions in the particle-hole $(p-h)$ and multipole pairing interaction in the particle-particle $(p-p)$ channels, respectively [20]. The model parameters are fixed empirically in such a way that the properties of the lowest-lying collective states and giant resonances are described accurately [23]. An exception is the isovector spin-dipole coupling constant, which is obtained from fully selfconsistent QRPA calculations using the microscopic EDF of Ref. [22].

The QPM formalism allows for a further expansion of QRPA $p-h$ excitations to multi-particle-multi-hole states in terms of coupling between quasiparticles and phonons [20]. Thus, for spherical even-even nuclei the model Hamiltonian is diagonalized on an orthonormal set of wave functions constructed from one-, two- and threephonon configurations [21]:

$$
\begin{aligned}
& \Psi_{v}(J M)=\left\{\sum_{i} R_{i}(J v) Q_{J M i}^{+}\right. \\
& +\sum_{\substack{\lambda_{1} i_{1} \\
\lambda_{2} i_{2}}} P_{\lambda_{2} i_{2}}^{\lambda_{1} i_{1}}(J v)\left[Q_{\lambda_{1} \mu_{1} i_{1}}^{+} \times Q_{\lambda_{2} \mu_{2} i_{2}}^{+}\right]_{J M} \\
& \left.+\sum_{\substack{\lambda_{1} i_{1} \lambda_{2} i_{2} \\
\lambda_{3} i_{3} I}} T_{\lambda_{3} i_{3}}^{\lambda_{1} i_{1} \lambda_{2} i_{2} I}(J v)\left[\left[Q_{\lambda_{1} \mu_{1} i_{1}}^{+} \otimes Q_{\lambda_{2} \mu_{2} i_{2}}^{+}\right]_{I K} \otimes Q_{\lambda_{3} \mu_{3} i_{3}}^{+}\right]_{J M}\right\} \Psi_{0},
\end{aligned}
$$

where $\mathrm{R}, \mathrm{P}$ and $\mathrm{T}$ are unknown amplitudes, and $v$ labels the number of the excited states.

The electromagnetic transition matrix elements are calculated for transition operators including the interaction of quasiparticles and phonons [24] where exact commutation relations are implemented which is a necessary condition in order to satisfy the Pauli principle. 
Table 1. Theoretical EDF+QPM results on neutron skin thickness $\delta r$, moments of the dipole photoabsorption cross section and the dipole polarizability of ${ }^{206} \mathrm{~Pb}$. Experimental data from $\left(\vec{\gamma}, \gamma^{\prime}\right)$ experiment at the $\mathrm{HI} \vec{\gamma} \mathrm{S}$ facility [4] and measurements of the photoabsorption cross section for $\mathrm{E}_{\gamma}>S_{1 n}$ reported in Refs. [26, 27] are shown in the 1st and 2nd rows.

\begin{tabular}{lllllllll}
\hline Nucleus & $\begin{array}{l}\delta r \\
(\mathrm{fm})\end{array}$ & $\begin{array}{l}\mathrm{E}_{\max } \\
(\mathrm{MeV})\end{array}$ & $\begin{array}{l}60 \mathrm{NZ} / \mathrm{A} \\
(\mathrm{mb} \mathrm{MeV})\end{array}$ & $\begin{array}{l}\sigma_{0} \\
(\mathrm{mb} \mathrm{MeV})\end{array}$ & $\begin{array}{l}\sigma_{-1} \\
(\mathrm{mb})\end{array}$ & $\begin{array}{l}\sigma_{-2} \\
(\mathrm{mb} / \mathrm{MeV})\end{array}$ & $\begin{array}{l}\alpha_{D} \\
(\mathrm{mb} / \mathrm{MeV})\end{array}$ & Refs. \\
\hline${ }^{206} \mathrm{~Pb}$ & & 26.4 & 2962 & $3544 \pm 294$ & $241.0 \pm 17.4$ & $17.6 \pm 1.4$ & $122.2 \pm 9.7$ & {$[4,26,27]$} \\
& & & & 3437 & 239.8 & 17.6 & 122 & {$[28]$} \\
& 0.15 & 25.0 & 2962 & 3060 & 230.4 & 18.3 & 127.0 & EDF+QPM
\end{tabular}
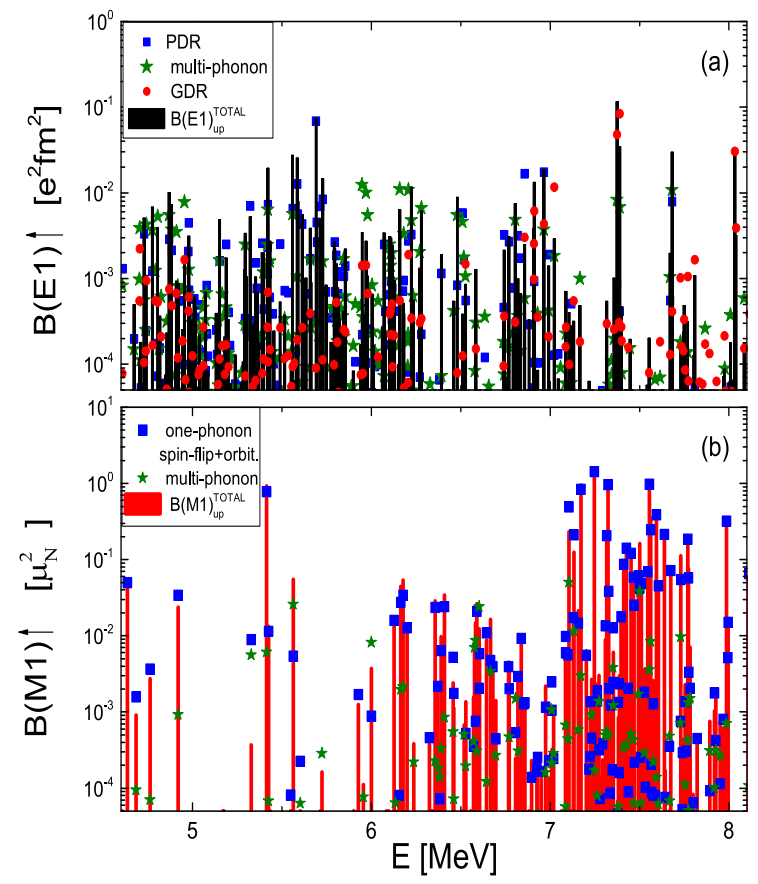

Figure 1. (Color online) Low-energy (a) electric dipole strength and (b) magnetic dipole strength distributions of ${ }^{206} \mathrm{~Pb}$ related to different counterparts of the transition matrix elements obtained from three-phonon EDF+QPM approach.

\section{Discussion}

Recently, much attention has been given to the understanding of the PDR [5]. This phenomenon is found to be a common feature in stable and unstable nuclei with neutron excess. Typically, the PDR is of electric character [10] and it appears as an additional dipole strength component around the neutron threshold sitting on top of the low-energy tail of the GDR classically represented by a Lorentzian shape $[11,25]$. Theoretical investigations of the dipole photoabsorption cross section $\sigma_{0}$ of ${ }^{206} \mathrm{~Pb}$ are performed in the frame of EDF+QRPA and EDF+QPM theory [4]. In the calculations both, E1 and M1 strengths up to $25 \mathrm{MeV}$ in ${ }^{206} \mathrm{~Pb}$ are taken into account. The obtained theoretical results of dipole photoabsorption cross section $\sigma_{0}$ and its higher moments $\sigma_{-1}$ and $\sigma_{-2}$ as well as energy-weighted-sum-rules (EWSR) are presented in Table 1 . In addition to the dipole photoabsorption cross section and its Lorentz extrapolation, a new low-energy structure of oscillating extra dipole strength at $\mathrm{E}_{\gamma} \approx 5-8 \mathrm{MeV}$ in ${ }^{206} \mathrm{~Pb}$ is found. Similar structure in the energy range
Table 2. Summary of the theoretical EDF+QPM results and data from $\left(\vec{\gamma}, \gamma^{\prime}\right)$ experiment at the $\mathrm{HI} \vec{\gamma} \mathrm{S}$ facility on E1 and M1 strengths in ${ }^{206} \mathrm{~Pb}$ taken from Ref. [4]. The experimental sensitivity limit for a single E1 transition is $\gtrsim 5 \times 10^{-4} \mathrm{e}^{2} \mathrm{fm}^{2}$ and for a single M1 transition is $\sim \times 10^{-2} \mu_{N}^{2}$, respectively.

\begin{tabular}{lcr}
\hline Parameter & Present data & EDF+QPM \\
\hline Energy interval (MeV) & $4.9-8.1$ & $4.9-8.1$ \\
Number of E1 states: & & \\
Within the exp. sensitivity & 100 & 94 \\
Total & & 340 \\
$\Sigma \mathrm{B}(\mathrm{E} 1) \uparrow\left(\mathrm{e}^{2} \mathrm{fm}^{2}\right)$ & $0.88 \pm 0.17$ & 0.935 \\
& & \\
Number of M1 states: & & 28 \\
Within the exp. sensitivity & 26 & 170 \\
Total & & 8.9 \\
$\Sigma \mathrm{B}(\mathrm{M} 1) \uparrow\left(\mu_{N}^{2}\right)$ & $8.25 \pm 1.97$ & \\
\hline
\end{tabular}

from about 7 to $11 \mathrm{MeV}$ has also been observed in our studies of $\mathrm{N}=50$ isotones [11]. The latter is related to PDR which is observed as well in other neutron-rich nuclei. It is explained as a vibration of the excessive neutrons against the symmetric $\mathrm{N}=\mathrm{Z}$ system. As an additional consequence, it is expected that the total PDR strength correlates with the ratio $\mathrm{N} / \mathrm{Z}$ [1].

Furthermore, from our EDF mean-field calculations we derive that the ${ }^{206} \mathrm{~Pb}$ nucleus exhibits a neutron skin with a thickness of $\delta r=0.15 \mathrm{fm}$ defined as a difference between the neutron and proton rms radii $\delta r=$ $\sqrt{\left\langle r^{2}\right\rangle_{n}}-\sqrt{\left.<r^{2}\right\rangle_{p}}$ (see also in Table 1). Accordingly, from EDF+QRPA calculations we observe a sequence of low-energy QRPA $1^{-}$states of almost pure neutron twoquasiparticle structure. The analysis of proton and neutron transition densities confirm further the dominant presence of neutron skin oscillations. Taking into account these considerations, the energy range below the neutron threshold of ${ }^{206} \mathrm{~Pb}$ could be associated with a genuine PDR mode.

Spectral distribution of low-energy E1 and M1 strengths below the neutron threshold in ${ }^{206} \mathrm{~Pb}\left(\mathrm{~S}_{n}=8.087\right.$ $\mathrm{MeV}$ ) are shown in Fig. 1. A summary of the obtained theoretical EDF+QPM E1 and M1 total strengths in comparison with data from $\left(\vec{\gamma}, \gamma^{\prime}\right)$ experiment at the $\mathrm{HI} \vec{\gamma} \mathrm{S}$ facility are presented in Table 2. It is found that the fragmentation of the E1 strength is due to the interaction of the QRPA PDR states with those from the GDR tail and also with multi-phonon configurations with different spin and parities. The detailed EDF+QPM analysis of the E1 transition matrix elements strongly suggests that the PDR dominates 
the distribution of the dipole strength up to about $7 \mathrm{MeV}$, at which point the tail of the GDR starts making an important contribution. Overall, the PDR and the GDR account for about $77 \%$ and $12 \%$ of the E1 strength below the neutron separation energy in ${ }^{206} \mathrm{~Pb}$, respectively. Also significant is the impact from multi-phonon states to the total E1 strength and to a lesser extent to the M1 strength. From the individual transitions displayed in Fig. 1, one may also compute experimental and theoretical cumulative strengths below the neutron separation energy and to visually assess the different spectral contribution to the overall dipole transition strength.

The theoretical cumulative electric and magnetic dipole strengths are compared to recent high-resolution $\left(\vec{\gamma}, \vec{\gamma}^{\prime}\right)$ experiment from the $\mathrm{HI} \vec{\gamma} \mathrm{S}$ facility in Fig. 2 which is also discussed in Ref. [4].

However, taking into account that the PDR contribution to the dipole photoabsorption cross section $\sigma_{0}$ is just a minor part of the order of a few percents, a more robust observable is the 'inverse-squared' energy weighted sum $\left(\sigma_{-2}\right)$. It can be defined with the relation [29]

$\alpha_{D}=\frac{1}{2 \pi^{2} \alpha} \int_{0}^{\infty} \frac{\sigma_{\gamma}(E)}{E^{2}} d E=\frac{\sigma_{-2}}{2 \pi^{2} \alpha}=6.942 \sigma_{-2}$,

where $\sigma_{-2}$ is directly proportional to the electric dipole polarizability $\alpha_{D}$ (both given in $\mathrm{mb} / \mathrm{MeV}$ ). From Eq. (4) we can calculate the total dipole polarizability $\alpha_{D}\left({ }^{206} \mathrm{~Pb}\right)(\mathrm{EDF}+\mathrm{QPM})=127 \mathrm{mb} / \mathrm{MeV}=18.3 \mathrm{fm}^{3} / \mathrm{e}^{2}$. The theoretical $\mathrm{EDF}+\mathrm{QPM}$ values are compared with data [4] in Table 1.

Further comparison of the $\alpha_{D}$ value in ${ }^{206} \mathrm{~Pb}$ with the one obtained in ${ }^{208} \mathrm{~Pb}$ [3] shows a decrease of $3 \%$ in ${ }^{206} \mathrm{~Pb}$. This is found correlated with the decrease of the neutron skin thickness of ${ }^{206} \mathrm{~Pb}$, which is $4 \%$ less than that of ${ }^{208} \mathrm{~Pb}$. It should be stressed that for the particular case of the dipole polarizability, the contribution of the strength beyond $\approx 25 \mathrm{MeV}$ is very small because of the $1 / E^{2}$ weighting of the photoabsorption cross section.

Needless to say, a proper description of the rich and complex experimental spectrum depicted in Fig. 1 requires a highly sophisticated theoretical treatment. A detailed description of the elaborate multi-quasiparticle and multiphonon approach also the technical aspects of the model could be found in Refs. [1, 6, 8, 10, 11, 13-16, 20].

The present analysis also shows that theoretical standard strength functions currently used for the calculation of cross sections in codes based on statistical reaction models do not describe the dipole strength distribution below the $(\gamma, n)$ threshold correctly and need to be improved by taking into account the observed enhanced strength (see also in Ref. [18]. In this aspect, the EDF+three-phonon QPM approach could be successfully implemented in statistical reaction codes to investigate neutron-capture cross sections of astrophysical importance [18, 19]. Such example is the our recent result on the neutron-capture cross section of the reaction ${ }^{85} \mathrm{Kr}(\mathrm{n}, \gamma){ }^{36} \mathrm{Kr}$ [19]. In these studies the microscopic EDF+QPM calculations are found in very good agreement with the experimental data on the one hand and the HFB+combinatorial results on the other hand [19].

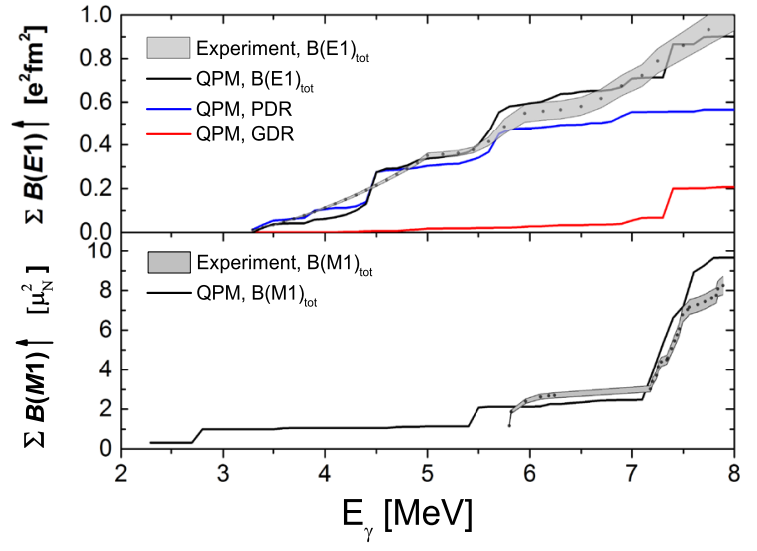

Figure 2. (Color online.) Cumulative $\mathrm{B}(\mathrm{E} 1)$ (top) and $\mathrm{B}(\mathrm{M} 1)$ (bottom) strength in ${ }^{206} \mathrm{~Pb}$ obtained from integrating the corresponding distribution of strength up to an energy $\mathrm{E}_{\gamma} \leq S_{1 n}=$ 8.087 MeV presented in Ref. [4].

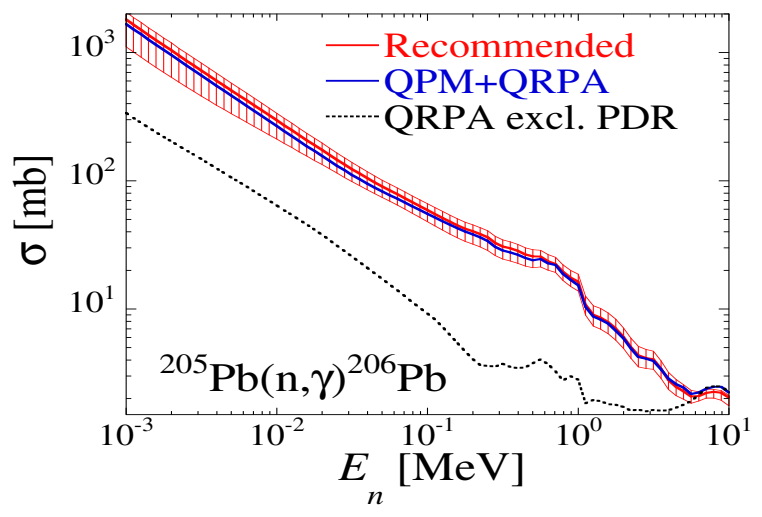

Figure 3. (Color online.) Radiative capture cross section ${ }^{205} \mathrm{~Pb}(\mathrm{n}, \gamma){ }^{206} \mathrm{~Pb}$ using as input the experimental $E 1$ and $M 1$ dipole strength (red curve) or/and the three-phonon EDF+QPM plus EDF+QRPA predictions (blue curve). The dotted line is obtained with the EDF+QRPA strength excluding the PDR contribution. See text and Ref. [4] for further details.

We now proceed to assess the impact of EDF+QPM calculations and the measurement from Ref. [4] on the neutron capture cross section in ${ }^{206} \mathrm{~Pb}$, and possibly on the stellar nucleosynthesis. The intensity and the energy distribution of the nuclear dipole response, including both the low-energy PDR plus the contributions from core polarization below the neutron separation energy are fundamental ingredients for the determination of the neutron-capture rates [18]. To estimate the radiative neutron cross section of ${ }^{205} \mathrm{~Pb}$, statistical model calculations using the TALYS1.8 code [30] have been carried out. The obtained results are displayed in Fig. 3 taken from our recent paper [4]. The "Recommended" curve corresponds to the radiative cross section obtained with the measured E1 and M1 $\gamma$ ray strengths from Ref. [4], complemented by EDF+QPM predictions outside the experimental energy range. The uncertainty band in Fig. 3 stems from the experimental uncertainties affecting the $\gamma$-ray strength, but also due to the use of different models to predict the nuclear level density [31, 32]. Finally, the recommended cross section is obtained with the combinatorial model of Ref. [33]. As shown in Fig. 3, the EDF+QPM model supplemented with 
QRPA calculations generate a cross section that is in excellent agreement with the predictions based on the experimental strength. The analysis of various individual contributions to the cross section: M1, PDR, and core polarization indicate a very strong impact of the PDR [4]. Furthermore, we observe that whereas the M1 contribution is rather insignificant (less than 5\%), the combined PDR plus core polarization contribution is crucial for a proper description of the cross section. Indeed, excluding the PDR contribution, QRPA predictions by themselves yield negligible E1 strength below $6 \mathrm{MeV}$, leading to a cross section about 5 times lower relative to the one involving the combined contribution. Based on these results, the experimentally constrained Maxwellian-averaged cross section at $30 \mathrm{keV}$ discussed in Ref. [4] is estimated to be $130 \pm 25 \mathrm{mb}-$ a value that is consistent with the prediction of $125 \pm 22 \mathrm{mb}$ estimated solely by theoretical means. This latter value has been recommended in Ref. [34] and has been traditionally used in s-process calculations. With this updated cross section, the dynamics involved in the survival and destruction of ${ }^{205} \mathrm{~Pb}$ in AGB stars is put on much more solid ground [4].

In conclusion, EDF+QPM calculations and high resolution $\left(\vec{\gamma}, \gamma^{\prime}\right)$ experiment at the $\mathrm{HI} \vec{\gamma} \mathrm{S}$ facility [4] are performed in the investigation of the electromagnetic dipole response of ${ }^{206} \mathrm{~Pb}$. The theoretical analysis permits a separation of the PDR from the tail of the GDR and multiphonon excitations due to core polarization effects. Our findings suggest that the low-energy dipole strength is predominantly electric in character and mainly due to a PDR skin oscillation. However, a substantial contribution from the low-energy tail of the GDR and multi-phonon states to the total E1 strength is also observed that is responsible for the fragmentation pattern of low-energy dipole states [4]. Moreover, the EDF+QPM theory successfully reproduces the low-energy M1 spectral distribution suggesting that it is mostly due to spin-flip excitations. The neutron-skin thickness in both ${ }^{206} \mathrm{~Pb}$ and ${ }^{208} \mathrm{~Pb}$ are provided, with the latter consistent with some recent analysis [3]. In the context of stellar nucleosynthesis, an updated experimentally constrained Maxwellian-averaged radiative capture cross section ${ }^{205} \mathrm{~Pb}(\mathrm{n}, \gamma){ }^{206} \mathrm{~Pb}$ is obtained [4]. The obtained theoretical results and the very good agreement with the experiment confirm the predictive power of the involved many-body theoretical method like the EDF+QPM approach for exploratory investigations of n-capture reaction rates in hitherto experimentally inaccessible mass regions.

\section{References}

[1] N. Tsoneva, H. Lenske, Phys. At. Nucl. 79, 885 (2016)

[2] J. Piekarewicz, Phys. Rev. C 73, 044325 (2006)

[3] A. Tamii et al., Phys. Rev. Lett. 107, 062502 (2011)

[4] A.P. Tonchev et al., Phys. Lett. B 773, 20 (2017)
[5] D. Savran et al., Prog. Part. Nucl. Phys. 70, 210 (2013)

[6] N. Tsoneva, H. Lenske, Ch. Stoyanov, Phys. Lett. B 586, 213 (2004)

[7] S. Volz et al., Nucl. Phys. A 779, 1 (2006)

[8] N. Tsoneva, H. Lenske, Phys. Rev. C 77, 024321 (2008) and refs. therein.

[9] R. Schwengner et al., Phys. Rev. C 78, 064314 (2008)

[10] A.P. Tonchev et al., Phys. Rev. Lett. 104, 072501 (2010)

[11] R. Schwengner, et al., Phys. Rev. C 87, 024306 (2013)

[12] Krishichayan et al., Phys. Rev. C 91, 044328 (2015)

[13] N. Tsoneva, H. Lenske, Phys. Lett. B 695174 (2011)

[14] L. Pellegri et al., Phys. Rev. C 92, 014330 (2015)

[15] M. Spieker et al., Phys. Lett. B, 752, 102 (2016)

[16] G. Rusev et al., Phys. Rev. Lett. 110, 022503 (2013)

[17] S. Goriely and E. Khan, Nucl. Phys. A 706, 217 (2002), and references therein.

[18] N. Tsoneva et al., Phys. Rev. C 91, 044319 (2015), and references therein.

[19] R. Raut et al., Phys. Rev. Lett. 111, 112501 (2013)

[20] V.G. Soloviev, Theory of complex nuclei (Oxford: Pergamon Press, 1976)

[21] M. Grinberg and Ch. Stoyanov, Nucl. Phys. A 573, 231 (1994)

[22] F. Hofmann and H. Lenske, Phys. Rev. C 57, 2281 (1998)

[23] A. I. Vdovin and V. G. Soloviev, Sov. J. Part. Nucl. 14, 237 (1983)

[24] V. Yu. Ponomarev, Ch. Stoyanov, N. Tsoneva, and M. Grinberg, Nucl. Phys. A 635, 470 (1998)

[25] B. L. Berman, At. Data Nucl. Data Tables 15, 319 (1975)

[26] T. Kondo, et al., Phys. Rev. C 86, 014316 (2012)

[27] R.R. Harvey, et al., Phys. Rev. 136, B126 (1964)

[28] Evaluated Experimental Nuclear Structure Data File, National Nuclear Data Center, Brookhaven National Laboratory, NY, (2016)

[29] O. Bohigas, N. Van Giai, D. Vautherin, Phys. Lett. B 102105 (1981)

[30] A.J. Koning, D. Rochman, Nuclear Data Sheets 113, 2841 (2012)

[31] A.J. Koning, S. Hilaire, S. Goriely, Nucl. Phys. A 810, 13 (2008)

[32] R. Capote, M. Herman, P. Oblozinsky, et al., Nuclear Data Sheets 110, 3107 (2009)

[33] S. Goriely, S. Hilaire, and A.J. Koning, Phys. Rev. C 78, 064307 (2008)

[34] Z. Y. Bao et al., Atomic Data and Nuclear Data Tables 76, 70 (2000) 